\title{
What predicts productivity? Theory meets individuals
}

\section{AUTHOR}

\section{AFFILIATION}

\begin{abstract}
Because they involve individual-level cognitive processes, psychological explanations of linguistic phenomena are in principle testable against individual behaviour. The present study draws on patterns of individual variation in corpus data to test explanations of productivity. Linguistic patterns are predicted to become more productive with higher type frequencies and lower token frequencies. This is because the formation of abstract mental representations is encouraged by varied types but counteracted by automation of high-frequency types. The predictions are tested for English -ly and -ness-derivation, as used by 698 individual journalists in the New York Times Annotated Corpus and 171 members of Parliament in the Hansard Corpus. Linear regression is used to model individual variation in productivity, in relation to type and token frequency, as well as several other predictor variables. While the expected effects are observed, there is also robust evidence of an ineraction effect between type and token frequency, indicating that productivity is highest for patterns with many types and not-too-infrequent tokens. This fits best with a view of entrenchment as both a conservative and creative force in language. Further, some variation remains irreducibly individual and is not explained by currently known predictors of productivity.
\end{abstract}

Keywords: derivational morphology; individual variation; productivity; token frequency; type frequency

\section{Introduction}

Patterns - or rules, or schemas, or constructions - underlie language's characteristic ability to produce an infinite variety of complex sequences. Yet, the patterns themselves are rarely if ever free from constraints. This is evident in derivational morphology (e.g. strong-strength, long-length, but wrong*wrength), but it arguably holds as well for inflectional morphology and syntax. The degree to which a pattern is capable of producing truly new complex sequences is its productivity. Just what determines a pattern's productivity has been a core area of research in morphology and syntax.

So far, our understanding of the determinants of productivity has been advanced by comparing across different patterns, measuring their productivity and correlating it to any other observable properties they have. However, as the determinants of productivity are now generally believed to reside in processing effects, which play out at the level of individual language users, it should be possible to test models of productivity also by taking a single pattern and comparing its productivity across individuals. This allows testing of predictions from the literature on independent data, and it can be expected to corroborate and even fine-tune the assumed psycholinguistic mechanisms underlying productivity.

From the literature, two of the main determinants of a pattern's productivity are its type frequency and, to a lesser extent, its token frequency. The former is held to correlate positively with productivity, the latter negatively. In the present study, the effect of these determinants is tested against the individual variation in productivity that can be observed in natural non-elicited usage, using 
multiple linear regression analysis. This is done for two morphological patterns in English: -ly-derivation, as in certainly, grammatically, yellowly, and -ness-derivation, as in flatness, correctness, Frenchness. The data is drawn from two independent sources: the New York Times Annotated Corpus and the Hansard Corpus. The results confirm previous claims. Type frequency proves to be the main determinant of productivity also across individuals, and effects are also observed for token frequency. However, the statistical models further indicate a more complex interplay between type and token frequency than could be demonstrated so far, showing a recurrent interaction effect between the two. This especially refines our understanding of the effect of token frequency, showing that token frequency actually promotes productivity provided it combines with high type frequency.

More generally, the findings invite some theoretical reflection on individual variation. On the one hand, the present study underscores the potential (but also some of the limits) of individual variation in natural usage as an alternative testing ground for psycholinguistic explanations of language structure. On the other, the findings also point to individual differences that are (as yet) irreducible. A significant portion of the variation between individuals is not explicable from our current theoretical models of productivity. Most strikingly, individuals' productivity scores for the two patterns investigated are found to be correlated, hinting at more fundamental individual differences in linguistic creativity. It must be concluded that while individual variation can support and refine current theoretical models, the theoretical models still underdetermine individual variation.

In what follows, an overview is given of previous work on productivity (Section 2). Next, the case study is presented, discussing its data, methods and general findings (Section 3). Finally, more thorough discussion of some of the findings is offered, relating them to the general model of productivity that has emerged from the literature, and reflecting on what they reveal about individual variation (Section 4).

\section{Determinants of productivity}

The literature on productivity is centred on a number of issues. First, there is the question whether productivity can be thought of as a single phenomenon, subject to the same principles, across derivational morphology, regular and irregular inflectional morphology, and syntax (see, for example, the debate between Rumelhart \& Mclelland 1987 and Pinker 1999). The present study addresses what is in this respect the least contentious area - derivational morphology. Therefore, no position can be explicitly argued for here, but in the following discussion I will draw indiscriminately from work in derivational morphology as well as in inflectional morphology and syntax, following the assumption that the differences between these domains are likely of a gradualist nature (Booij 2010: 89).

Second, some debate revolves around the question of what productivity is. Bolinger (1948: 18) defined productivity as "the statistical readiness with which an element enters into new combinations". The meaning of the term has not been stable, however. According to Barðdal $(2006,2008)$, who roughly echoes Corbin (1987: 177), productivity as it has come to be understood in the literature can be broken down into 'generality', 'regularity' and 'extensibility'. These subconcepts are jointly manifested in the most typical productive patterns, such as past tense -ed-suffixation in English, which is not subject to any semantic constraints (generality), has a perfectly predictable form (regularity), and is the pattern typically resorted to in analogical levelling or when new verbs are accommodated (extensibility). Essentially endorsing Bolinger's early definition, Barðdal further argues that "the REGULARITY 
and the GENERALITY subconcepts can be treated as derivatives of the EXTENSIBILITY concept of productivity" (2006: 469). This view is followed here, taking extensibility as the main component of what makes a pattern productive. In other words, a pattern is regarded as productive to the extent that it supports the creation of novel combinations.

What productivity is understood to be has obvious implications for how it should be measured. Taking extensibility as the core feature of productivity vies well with Baayen \& Lieber's (1991) early proposal to measure productivity in terms of the rate of occurrence of hapax legomena. Hapax legomena, or hapaxes for short, can be regarded as momentary extensions of a pattern. They are oneoff formations, attested in a corpus only once and therefore the instantiations of a pattern that can be most safely assumed to have been freshly 'computed' as true neologisms (drawing on a productive rule), rather than having been 'retrieved' from lexical storage as ready-made units (without relying on a productive rule). Slightly adapted measures have been proposed by Baayen (1993), but as far as the extensibility component of productivity is concerned hapaxes have remained the essential touchstone (see the discussion in Plag 1999: 32-35).

Note, nevertheless, that hapaxes are not completely unproblematic. Even though their one-off character gives hapaxes a privileged status over merely low-frequency types, it should be borne in mind that what counts as a hapax is still relative to corpus size. Many hapaxes in a small corpus may not be hapaxes in a larger corpus and many hapaxes in a larger corpus will not be attested in a smaller corpus. This can cause difficulties when dealing with unusually big or unusually small data sets. The bigger the corpus, the more exotic the hapaxes, to the point where hapaxes predominantly include bizarre formations bordering on ungrammaticality. The smaller the corpus, the greater the risk that hapaxes include types with moderate-to-high frequencies in the population. This problem will have to be returned to below when productivity is operationalized for the purpose of the present study.

Third, most central to this paper there is the question what determines productivity. Here, several factors have been argued to come into play. For example, the number of potential bases for a morphological process may be finite; there may be semantic or phonological constraints on the application of a pattern; new formations may be more or less useful from a pragmatic point of view; or a pattern may suffer from competition with another pattern producing roughly synonymous forms (Plag 1999: ch.3).

The main determinants of productivity, however, have been argued to reside in processing effects that revolve around a pattern's type and token frequencies. On the one hand, productivity increases with a pattern's type frequency (Plag 2003; Barðdal 2006; Goldberg 2006; Bybee 2007; Schmid 2017). The reason is believed to be that the occurrence of varied manifestations of a pattern encourages and enables language users to abstract away from the concrete forms they directly encounter. In doing so, they begin to form more general or schematic representations. This schematization process in turn is what underlies productivity because it creates representations with the kind of open filler slots that can eventually attract new fillers. On the other hand, a pattern's token frequency can detract from productivity (Baayen \& Lieber 1991; Plag 2003; Bybee 2007). The reason is thought to be that high-frequency forms tend to be stored autonomously and to be processed as whole units. This makes high-frequency forms "less likely to participate in schemas", keeping them from contributing to schema formation (Bybee 2007: 174). A pattern with many high-frequency types will therefore be less productive. Plag (2003: 54) summarizes the effects of type and token frequency on productivity as follows: 
Each time a low-frequency complex word enters the processing system, this word will be decomposed, because there is no whole-word representation available. This decomposition will strengthen the representation of the affix, which will in turn make the affix readily available for use with other bases, which may lead to the coinage of new derivations. If, however, only highfrequency complex words enter the system, there will be a strong tendency towards wholeword storage, and the affix will not be so strongly represented; it will therefore not be so readily available for new formations.

In addition to the effects following from a pattern's type and token frequencies, several other processing effects have been argued for. For example, Plag (1999: 38) suggests that speakers may infer a pattern's productivity by monitoring their environment: if they do not come across transparent lowfrequency types they may conclude that a pattern is not productive and may refrain from using it productively themselves. A very similar idea has been developed further by Goldberg (2006) and Stefanowitsch (2008), who speak of 'negative entrenchment' (see also Goldberg this volume). Further, Hay (2003) offers a subtler interpretation of the effects of token frequency, arguing (for derivational morphology) that autonomous storage will be dependent on the ratio of a derived form's frequency to the frequency of its base. Hay \& Baayen (2002) follow up on this by showing that a pattern will be the more productive the smaller its share of types that are more frequent than their bases.

\section{Productivity across individuals}

To be truly persuasive, explanations of linguistic phenomena that resort to psychological principles should be able to make predictions about individual behaviour, to be tested either under experimental conditions or simply through observation of individuals' natural non-elicited behaviour. To the extent that they are linked to processing effects, this should be true also of several of the factors believed to determine a linguistic pattern's productivity. There is no escaping Booij's (2010: 93) suggestion:

It is possible that individual language users differ considerably in the degree to which they develop subschemas for the coinage of complex words, since they differ in their lexical knowledge and in the degree to which they create new complex words.

But is it also possible to test this systematically? Can we draw on our current understanding of productivity in order to predict how productive a pattern will be for an individual, given what we know about other aspects of that individual's linguistic behaviour? From the literature surveyed above, we can reasonably expect that a speaker who has many types for a pattern in their linguistic repertoire will be more likely to produce novel types of the pattern than a speaker who has few, when token frequency is held constant. Similarly, we can expect that a speaker who has many tokens of a pattern will use the pattern less productively than one who has few, when type frequency is held constant.

To test such expectations, individual variation in productivity is to be statistically modelled here for two derivational patterns, -ly and -ness-derivation. Individual variation will be investigated naturalistically, using corpus data. The following first describes the two patterns in the aggregate data (Section 3.1), next discusses the corpora used for the analysis (Section 3.2), then turns to the operationalization 
of dependent and independent variables for the individual variation study (Section 3.3) and finally reports the results of the linear regression analyses modelling the variation in productivity across individuals (Section 3.4).

\section{1 -Ly and -ness-derivation}

-Ly-derivation derives adverbs from (mostly) adjectives; -ness-derivation derives abstract nouns from (mostly) adjectives. Both patterns are at the extreme end of derivational productivity. Regarding -lyderivation, the question has been raised whether -ly might not be better regarded as an inflectional affix (Plag 2003: 97-98; Giegerich 2012). As for-ness-derivation, -ness has been listed as "perhaps the most productive suffix of English" by Plag (2003: 92).

Even so, the patterns' applicability is not completely indiscriminate or automatic. For example, regarding - $/ y$-derivation, it is rare to find -ly-adverbs derived from adjectives ending in /li/, as in (1a-b). Presumably on pragmatic grounds, -ly-adverbs are also seldom formed from certain semantic classes of adjectives, such as colour terms or adjectives describing spatial properties or configurations, as in (2). -Ness-derivation strongly favours gradable adjectives and tends to impose gradability on any base that would itself favour a non-gradable reading, as can be seen in (3). Further, some possible -nessforms remain (almost) unattested because their formation is in most contexts blocked by a betterentrenched alternative, such as youth for youngness or rarity for rareness: the blocked forms can occur, as (4) shows, but their use is exceptional. Finally, both -ly-derivation and-ness-derivation typically select adjectival bases, but still show some freedom to be applied to other types of bases as well, including bases whose adjectival status is somewhat questionable, as in (5a-b) as well as bases that are evidently nouns, as in (5c-d).

(1) a. I said, 'You know, Saul, you've been a profound influence on me.' He looked at me chillily and said, 'I would be surprised if I wasn't.' (NYTAC)

b. As the House knows well, we have already put in place what are laughingly, or friendlily, called the SAMs - sanctions assistance missions. (HC)

(2) a. If you want to understand the man - the comic genius, the author of the blackly buoyant and sorely missed "Far Side" comic strip, and a cartoonist so revered among scientists that they have named a louse and a butterfly after him - then look at his work. (NYTAC)

b. It happens to be regrettable, but it cannot be discredited, neither largely nor "smally" - if that is the right word. (HC)

(3) a. We should cherish the publicness of public schools; the ultimate goal of all reform is to make public schools work for everyone. (NYTAC)

b. It was in Moscow that I began to ponder the Europeanness of Russia, a difficult but essential subject. Where does Europe end? (HC)

(4) a. It's time that the youngness of our pitching staff is done and over with (NYTAC)

b. However, the rareness and exceptional nature of the cases should not obscure the growing significance of this problem. (HC)

(5) a. Although bosons cozy up to one another without complaint, fermions, which include electrons and protons, are quantumly antisocial. (NYTAC)

b. I have said that we should take some of their expertness with a pinch of salt. (HC) 
c. the Sound [i.e. the estuary north of Long Island] is so nutriently enhanced, you're selecting for special species that can use that high organic input (NYTAC)

d. I shall judge the true Labourness of the Government on what they do for ordinary families (HC)

From the various soft constraints on -ly-derivation and -ness-derivation it should be clear that there is ample room for linguistic creativity in the use of these patterns, as (1)-(5) indeed illustrate. It also follows that there is room for variation in how productively these resources are used by individuals.

\subsection{The data}

For an observational study of individual variation to be possible, enough spontaneously produced data is needed for a big enough group of individuals that is ideally not too heterogeneous with respect to other parameters known to affect variation. This is a tall order and finding the right type of data will presumably remain a serious obstacle for individual variation studies for some time to come. However, problems can be mitigated by seeking convergent evidence from independent sources, which is why this study draws on two independent data sets. The first is the New York Times Annotated Corpus, which contains all issues of the New York Times over a 21-year period from 1987 to 2007, amounting to about 1.13 billion words of text. The second is the 1990-2005 portion from the proceedings of the British House of Commons, as available through the Hansard Corpus. This part of the Hansard Corpus contains approximately 148 million words. The two corpora, henceforth NYTAC and $H C$, can be thought of as representing two loosely structured communities of language users with broadly similar communicative goals and linguistic practices. Both are sizeable enough and contain the required metadata to study individual variation.

Nevertheless, both corpora also have their problems. Though undoubtedly an idealization, it is useful at this point to distinguish between lectal and diatypic variation (following Gregory 1967). Lectal variation involves variation tied to generally stable properties of the speaker, such as their gender, age, locality, class etc. Lectal variation is of course present also in the NYTAC and HC, but it is assumed here that this is not a major cause for concern. Although it would be difficult, perhaps impossible, to disentangle purely idiolectal variation from other types of lectal variation, whatever unique or not-so-unique constellation of lects a speaker or writer represents, their individual lectal profile should reflect a relatively stable and coherent internalized system. For present purposes, it does not matter whether the differences between individuals are due to their gender, age or place of birth or indeed purely individual, as long as those differences credibly reflect ingrained speech habits. It is for this reason that I avoid the term 'idiolect' when speaking of individual variation, but I have made no attempt here to introduce additional lectal dimensions into the analysis.

Diatypic variation, in contrast, is the variation that comes from aspects of the communicative setting to which language users adjust, such as mode, genre, register, topic, etc. The NYTAC data, in particular, shows considerable internal heterogeneity on the diatypic dimension. A newspaper such as the New York Times contains a whole range of text types, from editorials to news items, to reviews, to columns, to obituaries, as well as covering an enormous variety of topics, including sports, politics, lifestyle, science, music, etc. This in itself would not be too problematic were it not that in this textual landscape each of the journalists contributing to the newspaper has their own more or less unique 
niche, meaning that individual differences may be hard to disentangle from diatypic variation. This is troublesome, because diatypic variation is less clearly reflective of ingrained speech habits. Intuitively, one can be a speaker of New Zealand English, but one cannot be a speaker of editorial English. The reason is that diatypic variation is exactly the dimension of variation on which speakers retain the greatest freedom to adjust their speech habits. For that reason, an attempt is made here to keep diatypic variation under control, in as far as the NYTAC allows, by incorporating some diatypically relevant information in the analysis (see below).

For the $H C$, the main issue is the complicated transmission history of its texts, which have presumably been prepared before being delivered in Parliament (not necessarily by the same person) and have then been transcribed and edited (involving several third parties) into the format found in the Parliamentary proceedings. It is far from clear, then, that the recorded speeches represent a single authorial voice. In the terminology introduced above: there is a potential 'overlay' of different individual lectal profiles. Mollin (2007), for example, finds that in addition to losing some of the performance characteristics of spoken interaction, the $\mathrm{HC}$ transcripts tend to opt for more conservative grammatical and lexical variants than were used by the actual speakers. That this complex transmission process introduces some noise into the data is evident. Still, while this is less than ideal, it is difficult to see how any signal of individual variation that can be picked up in our analysis could be an artefact of the transmission process.

To be sure, the problems of internal heterogeneity and textual transmission are not unique to the NYTAC and the $H C$ respectively. Third-party intervention in the textual transmission process must also affect the NYTAC material, though probably rather less severely. One mitigating factor here is the high time pressure under which newsrooms operate (as is reflected in the higher incidence of typing errors in the NYTAC compared to the $H C$ ), precluding invasive editing. Another is the fact that, in contrast to the $H C$ data, there is no shift in medium or intended audience during transmission. Similarly, some degree of internal heterogeneity can be expected in the $H C$ material, but with less individual divergence. More precisely, while individual members of Parliament may have specific areas of expertise or may have specialized roles in Parliament, they will in principle all engage in the same debates. Moreover, each member represents a constituency, which will inevitably see them address a very mixed bag of issues and concerns. In this light, the NYTAC and HC can be thought of as complementary, both being somewhat deficient as data sources but at least being deficient in different ways.

From the NYTAC and HC, data has been collected for all individuals that produced 250,000 words of text or more. Meeting this criterion are 698 New York Times journalists and 171 members of the House of Commons. To facilitate the comparison of counts for types and hapaxes (see below), and to ensure that individuals are equally represented in the aggregate data, all individuals contributed exactly 250,000 words of text to the data set that was ultimately retained for analysis. Since the data is chronologically structured, the material selected to represent any individual consists simply of the first 250,000 words they produced within the period chosen for study. The figures reported below are based on these sub-corpora, totalling 174.5 million words (from NYTAC) and 42.75 million words (from $H C$ ) respectively. To avoid confusion, the sub-corpora will be referred to as $N Y T A C_{S}$ and $H C_{S}$ (with $S$ for sub).

From NYTAC $C_{S}$ and $H C_{S}$, all words have been retrieved ending in -ly and -ness. False positives have been removed manually, going through the lists of types found in each corpus and checking tokens in context in case of doubt. False positives include any words orthographically ending in -ly or -ness but 
not being derivationally complex (butterfly, Sicily, harness, McWellness) or belonging to a different derivational pattern (girly, monthly, fatherly, governess). False positives also include any misspellings of otherwise legitimate types (embarrssingly, unwilllingness). The exclusion of misspellings is to avoid distorting type frequency counts (the effect on token frequency counts is negligible). What results is the token and type frequencies for -ly and -ness-derivation detailed in Table 1. The table also shows the number of hapaxes per derivational pattern per corpus, as well as the number of low-frequency types, defined as types with a frequency below 0.1 occurrence per million words ( $p m w)$. The large share of hapaxes and low-frequency types testifies to language users' ability to productively draw on -ly and -ness-derivation to create unique or very rare types.

Table 1. Aggregate frequencies for tokens, types, low-frequency types $(<0.1 \mathrm{pmw})$ and hapaxes for $-l y$ and -ness-derivation in NYTAC and $H C_{S}$.

\begin{tabular}{lccccrr} 
& TOKENS & TYPES & TYPES $<0.1$ PMW & HAPAXES & \\
\hline \multirow{2}{*}{ - Ly-derivation } & $1,553,728$ & 4,373 & 2,687 & 953 & $N Y T A C_{S}$ \\
\cline { 2 - 5 } & 441,131 & 2,116 & 930 & 513 & $H C_{S}$ \\
\hline \multirow{2}{*}{-Ness-derivation } & 87,880 & 2,256 & 1,753 & 685 & $N Y T A C_{S}$ \\
\cline { 2 - 6 } & 17,297 & 624 & 407 & 250 & $H C_{S}$ \\
\hline
\end{tabular}

\subsection{The variables}

In order to model variation in the productivity of -ly and -ness-derivation across individual language users, the following variables have been set up, all describing aspects of an individual's usage profile. The dependent variable is an individual measure of PRODUCTIVITY for each derivational pattern. The main independent variables are TYPE FREQUENCY and TOKEN FREQUENCY, again for each pattern. A number of additional variables have been introduced, for various reasons. These include MAIN DESK, a variable intended to capture some of the diatypic variation in NYTAC ; and LEXICAL DIVERSITY and GENERAL PRODUCTIVITY, two variables meant to capture more general aspects of individuals' linguistic profile. The following discusses how these variables have been operationalized. For clarity, note that the relation between these variables will be modelled in four separate regression analyses - one per sub-corpus and one per derivational pattern.

Not all of the determinants that potentially influence productivity, as discussed above (see Section 2), have been operationalized. Some are simply unlikely to be of interest here because they do not relate to the processing effects that play out on the level of individuals. Probably the best candidate for inclusion in follow-up work is the share of derived types that are more frequent than their bases, as proposed by Hay \& Baayen (2002). In principle, this could be measured for every individual in the two corpora. However, since this determinant can be expected to correlate with TOKEN FREQUENCY and would prove difficult to implement in practice (see AUTHOR \& OTHER (YEAR), who implemented it in a corpus-based priming study on the analysability of -ly-derivations), it has not been included here.

3.3.1 Productivity (P): An easily applicable measure for productivity is that proposed by Baayen \& Lieber (1991), which takes the ratio of hapaxes over tokens. However, this measure has been primarily intended for comparison of productivity across derivational patterns with vastly different token fre- 
quencies, which is not the case here. Moreover, incorporating token frequency in the dependent variable would defeat the point of testing the impact of token frequency on productivity as an independent variable. It therefore makes more sense to simply count hapaxes. All individuals contribute exactly 250,000 words of text to the corpus anyway, meaning they have, so to speak, all been given the same opportunity to produce any number of hapaxes.

The next problem, however, is how to define a hapax. Should hapaxes be identified as types with one occurrence in the usage of an individual (regardless of their frequency in the rest of the corpus), or as types with one occurrence in the aggregate data set? The first option appears to be more true to the ideal of taking individual grammars as the unit of analysis, but there would be a serious downside to it. For example, the forms effectiveness, competitiveness, illness and willingness have all been used exactly once by the same speaker in $H C_{s}$. But treating them as hapaxes would be to lose the value of hapaxes as unique on-the-fly formations. In fact, the four types in question are all among the 10 most frequent -ness-forms in $H C_{s}$, rendering it unlikely that the speaker in question had coined these forms productively, rather than simply retrieving them from lexical memory. The second option is also problematic. First, because NYTAC and $H C_{S}$ are very large data sets, many aggregate-level hapaxes are conspicuously unusual formations (e.g. yawningly, Teutonically, fertilely, Xavierness, horsiness, carnivorousness, etc.). What the number of aggregate-level hapaxes in an individual's usage would reflect might therefore end up being more akin to that individual's linguistic playfulness than to their more general readiness to extend a pattern to new linguistic contexts, beyond the combinations they have stored. Second, a large share of individuals would go down the record with no hapaxes at all, implying that - $/ y$ - and ness-derivation are unproductive for the majority of speakers of English. Even if we must not perhaps dismiss that possibility out of hand, it would be counterintuitive. On a practical note, moreover, having large numbers of individuals with zero productivity would lead to an extremely skewed distribution of productivity across the population, which is undesirable for modelling purposes.

The solution eventually adopted here is to measure PRODUCTIVITY as the number of types produced by an individual with only a single occurrence in the individual's usage and a frequency below $0.1 \mathrm{pmw}$ in the aggregate data set (compare Table 1 above). To give an idea of what this frequency threshold means, $0.1 \mathrm{pmw}$ corresponds to an average rate of one occurrence per year in the records of the British House of Commons between 1990 and 2005. This operationalization keeps PRODUCTIVITY from being too exclusively based on playful or deliberately conspicuous formations. But it also prevents it from being unduly influenced by types that actually have high token frequencies in the aggregate data, or types that have low aggregate token frequencies but high token frequencies in a particular individual. For example, nearly, which is the third most frequent -ly-adverb in NYTAC, does not contribute to the productivity measure for the one individual who uses it only once. Conversely, creamily, which occurs 12 times in NYTACs, giving it a normalized frequency below $0.1 \mathrm{pmw}$, does not contribute to the productivity measure of the one individual who uses the adverb 9 times and so for whom it is probably a stored form. This operationalization further ensures that the distribution of values for PRODUCTIVITY approximates a normal distribution. Finally, PRODUCTIVITY remains close to the original philosophy underlying the reliance on hapaxes as the tell-tale signs of a productive pattern. If someone uses a complex form only once and that form has very low frequency among their peers, it is unlikely that they could have retrieved the form from lexical memory, meaning the form has probably been productively coined. 
3.3.2 TOKEN FREQUENCY (TOKF) and TYPE FREQUENCY (TYPF): TOKEN FREQUENCY and TYPE FREQUENCY correspond respectively to the number of tokens and types for a given pattern for a given individual. The types contributing to the productivity measure have been subtracted from individual token and type frequencies, so as to avoid incorporating the dependent variable in the independent variables. The motivation for including token and type frequency in the model are obvious from the literature on productivity, which predicts a positive effect for type frequency and hints at a negative effect for token frequency (see Section 2 above).

3.3.3 MAIN DESK (MD): Introduced for the NYTAC $S$ material only, MAIN DESK is the only categorical variable in the analysis. As pointed out above, any issue of the New York Times is actually a collection of diverse text types, with journalists specializing in only a subset of topics and genres. It is likely that this influences journalists' productive usage of -ly and ness-derivation, independently of the more systeminternal determinants of productivity. For example, New York Times news coverage adopts a deliberately disengaged style that is in contrast to the often more playful, erudite and personal style found in its pieces on culture or lifestyle. Such differences in style may encourage or discourage the use of more far-fetched coinages, thereby potentially influencing PRODUCTIVITY. The NYTAC metadata contain information that can be used to control for this type of variation to some extent. Specifically, every article in the corpus is tagged for the desk in The New York Times newsroom that produced it. Some of the most common desk tags in NYTAC include the 'metropolitan desk', 'foreign desk', 'national desk', 'sports desk', 'financial desk', 'editorial desk' and 'cultural desk'. As can be seen from this, the desk tag contains information about the topic of an article, and the desk is also related (but not identical) to the newspaper section in which the article appeared.

Because most individuals in NYTACs do not contribute to a single desk, the variable MAIN DESK supplies the desk to which they mainly contribute. More precisely, for a given individual, MAIN DESK is the desk label assigned to more than half of their textual output, measured in number of words. As there are many desk labels in NYTAC, some groupings have been made prior to determining MAIN DESK. 'News' includes the metropolitan, national and foreign desks. 'Sports' corresponds to the sports desk. 'Finance' corresponds to the financial desk, but also including any mixed desk labels (e.g. 'business/financial desk'). 'Culture and lifestyle' includes the cultural desk, arts and leisure desk, book review desk and weekend desk, as well as any mixed labels containing any of these. 'Other' groups the remaining desks. MAIN DESK is also given the value 'other' when no main desk can be identified (i.e. because no single desk label covers more than half an individual's output). The groupings have been chosen to ensure that the value for MAIN DESK captures a degree of topical and stylistic homogeneity while avoiding a proliferation of desk labels. With the current grouping, only 153 individual journalists in NYTACS (22\%) had to be assigned to the least informative category of 'other'. 'News' is the most common category, with 289 journalists (41\%) assigned to it. Individuals with 'news' as main desk also tend to be the least productive users of -ly and -ness-derivation, which is why 'news' has been set as the reference level in the regression models presented below.

3.3.4 LEXICAL DIVERSITY (LD): Given the way the dependent variable PRODUCTIVITY has been operationalized, it can be expected that individuals with richer vocabularies may score higher on PRODUCTIVITY. This is because such individuals have access to more potential bases to apply derivational processes to. For this reason, a measure of lexical diversity has been added to the variables. LEXICAL DIVERSITY has been 
calculated by sequentially dividing every individual's 250,000 words of text into 250 batches of 1,000 words, automatically counting the number of word types in every batch and then taking the average type count. This has been preferred over the alternative of simply counting all word types in the full set of 250,000 tokens. The latter procedure, while giving more weight to low-frequency types, would also have been far more sensitive to spelling errors, which given the size of the data set it would have been impossible to remove manually.

3.3.5 GeNERAL PRODUCTIVITY (GENP): Preliminary inspection of the data reveals that individuals' scores for PRODUCTIVITY correlate across - $/ y$ and -ness-derivation, with a correlation coefficient of 0.50 in $H C_{S}$ and one of 0.82 in NYTACs. In other words, it is generally the same individuals that score high on PRODUCTIVITY for -ly-derivation as do for-ness-derivation. This could point to something like a more general individual propensity for using morphological patterns more or less productively, but it could also be an artefact produced by determinants of PRODUCTIVITY that are shared across -ly and -nessderivation, such as the variables operationalized as MAIN DESK and LEXICAL DIVERSITY. To at least begin to explore the issue, the variable GENERAL PRODUCTIVITY is introduced. When modelling the individual variation in the PRODUCTIVITY for -ly-derivation, GENERAL PRODUCTIVITY corresponds to the PRODUCTIVITY values for-ness-derivation and vice versa.

\subsection{Results}

Four linear regression models have been set up with the variables specified above, one per sub-corpus and per derivational pattern. They will be referred to here as Iy_NYTAC $C_{s}$ ness_NYTAC $C_{s}, I y_{-} H C_{s}$ and ness_HC. To facilitate interpretation of the model coefficients, as well as comparison across the models, all continuous variables have been standardized to a distribution with mean 0 and standard deviation 1. All models have been run with a bidirectional variable selection procedure. High leverage for a few data points motivates removal of one individual from Iy_NYTAC $C_{S}$ and two from ness_NYTAC . Model quality is not perfect, despite moderately to very acceptable Adjusted $R^{2}$ values from 0.36 for ness_HC to 0.87 for ness_NYTAC , and very low p-values for the F-statistics. Specifically, VIF values, though all below 10, indicate some risk of multicollinearity especially for ness_NYTAC . Moreover, Iy_NYTAC $C_{S}$ and ness_NYTAC $C_{S}$ suffer from heteroscedasticity. In that light, it is probably safe to take the high $\mathrm{R}^{2}$ values for $I_{-}$NYTAC and ness_NYTACS with a grain of salt, while $l_{-} H C_{S}$ is arguably the most reliable model, with a sound adjusted $\mathrm{R}^{2}$ of 0.59 and no compromising issues. Detailed model outputs are given in the Appendix.

The main interest of the models lies in their comparison, which gives a remarkably consistent picture. To show this, Figure 1 visualizes the coefficients for the various independent variables retained in the model, along with their $95 \%$ confidence intervals. Variables with a negative coefficient correlate negatively with the dependent variable PRODUCTIVITY; variables with a positive coefficient correlate with it positively. If a coefficient's confidence interval overlaps with zero, its variable has no significant effect in the model. As the variables have been standardized, a coefficient of 1 can be read as predicting an increase of one standard deviation in the dependent variable for an increase of one standard deviation in the predictor, while holding all other predictors constant. 


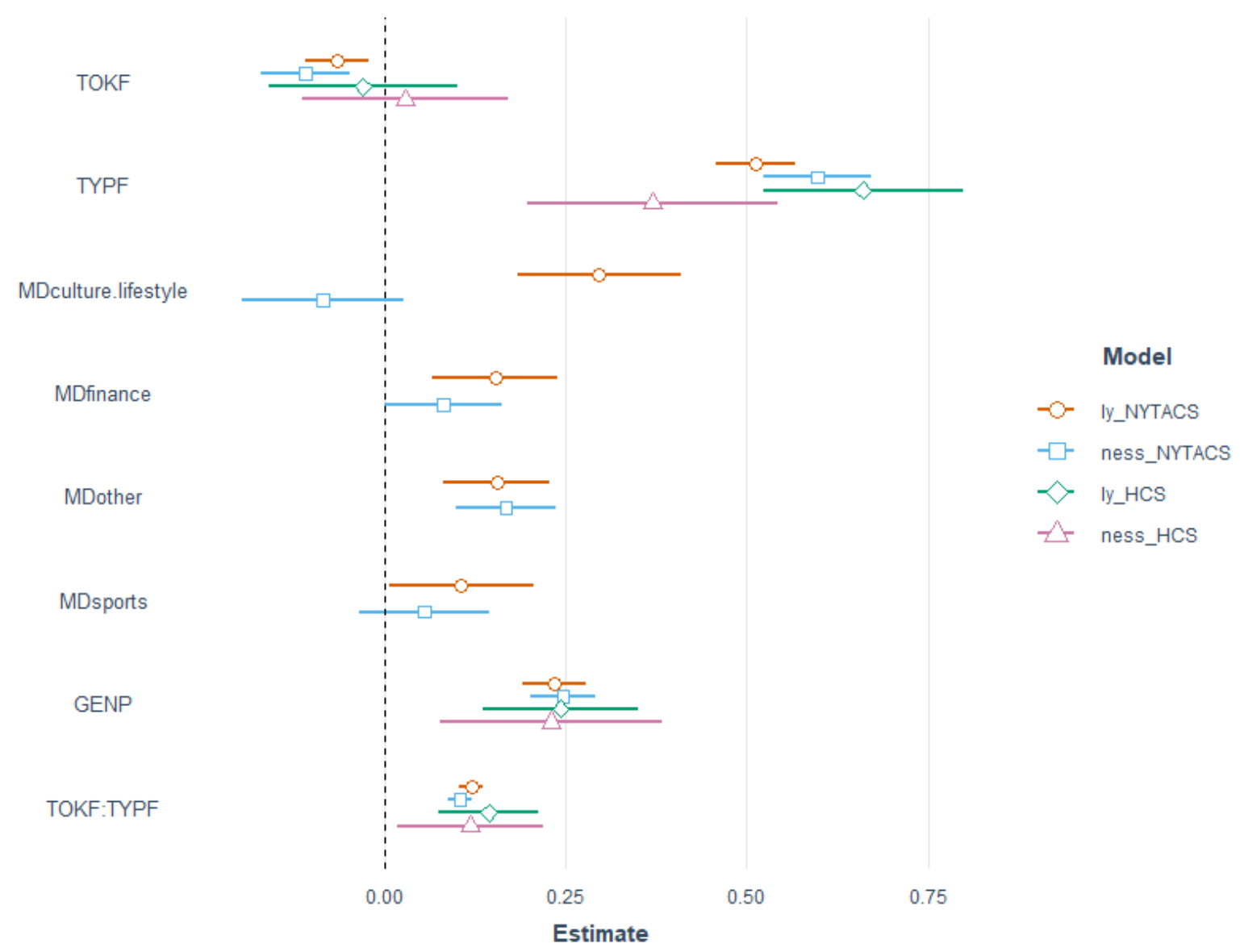

Figure 1. Variable coefficients with $95 \%$ confidence intervals for significant effects in four linear regression models (plot created with jtools).

As predicted by the literature, the strongest effect on PRODUCTIVITY comes from TYPE FREQUENCY, with the expected positive correlation found in all four models. The expected negative effect for TOKEN FREQUENCY is also evidenced, if less strongly, with negative effects in ly_NYTAC $C_{S}$ and ness_NYTAC , but no effect in $l y \_H C_{S}$ or ness_HCs. Consistently across the four models, however, there is a positive interaction between TOKEN FREQUENCY and TYPE FREQUENCY, to be discussed at greater length below. Specifically for the NYTAC data, MAIN DESK adds to explaining some of the variation, showing that journalists associated with the 'news' desks (the reference level in the regression models and therefore not displayed) tend to use -ly and -ness-derivation less productively, as well as hinting at a difference for the 'culture and lifestyle' desks between -ly and -ness-derivation. The initial observation that individual productivity is positively correlated across -ly and -ness-derivation survives in the regression analyses, with significant positive effects for GENERAL PRODUCTIVITY in all four models, a finding also returned to below. Finally, LEXICAL DIVERSITY is not plotted in Figure 1 because it is not retained as a predictor by the stepwise variable selection procedure in any of the models. When the models are run without stepwise variable selection, lexical diversity is reported with no significant effect.

\section{Discussion}

What can we take away from this? I will turn first to what the above findings tell us about productivity, focusing on how they relate to our current understanding of the determinants of productivity (Section 
4.1). Next, the question is addressed what the findings tell us about individual variation as a newly emerging field of study (Section 4.2).

\subsection{Understanding productivity}

At least in the present case, there appears to be a good overall match between what general linguistic theory predicts and how individual language users behave. The generalization that the productivity of a pattern benefits from high type frequency but suffers under high token frequency is supported by the behaviour of individuals, even if the evidence for the negative effect of token frequency remains somewhat more tentative.

At the same time, the results from the present individual variation study point to a more complex interplay between type and token frequency. The interaction found between the two implies that, on the one hand, the negative effect of token frequency on productivity becomes more pronounced when type frequency is low, but can be neutralized or even reversed when type frequency is high. On the other, the effect of type frequency is boosted when token frequency is high, while it is softened when token frequency is low. Illustrating this, Figure 2 shows the two effect plots for the interaction between TYPE FREQUENCY and TOKEN FREQUENCY for $l y \_H C_{S}$ (chosen here on the assumption that it is the most reliable of the four models - see above). The left panel shows the value of PRODUCTIVITY as predicted by TOKEN FREQUENCY, when TYPE FREQUENCY is either at its mean value (0), one standard deviation above its mean value (1) or one standard deviation below its mean value (-1). The right panel shows the value of PRODUCTIVITY as predicted by TYPE FREQUENCY, when TOKEN FREQUENCY is either at its mean value (0), one standard deviation above its mean value (1) or one standard deviation below its mean value $(-1)$.
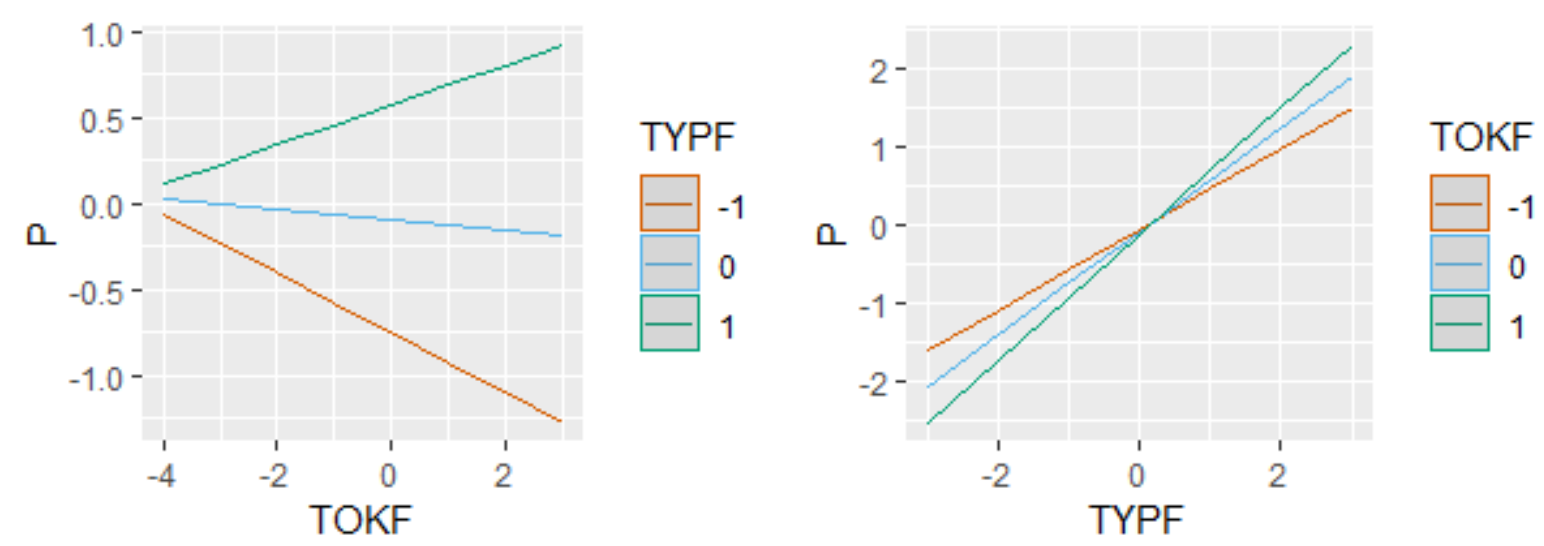

Figure 2. Effect plots for the interaction between TOKEN FREQUENCY (TOKF) and TYPE FREQUENCY (TYPF) as affecting predicted values for PRODUCTIVITY (P) in $I_{-} H C_{S}$ (plots created with sjPlot).

The interaction between type and token frequency makes theoretical sense. Recall that the reason type frequency is expected to promote productivity is that high type frequency encourages schematization and schematization is a necessary condition for productivity. The reason token frequency is expected to detract from productivity is that a pattern with many high-frequency types is likely to 
have many highly automated types which, being stored autonomously, do not contribute to schematization.

However, while autonomous storage is a well-documented effect of token frequency it is not the only effect. Repeated use of or exposure to a pattern leads to strong entrenchment of that pattern, which correlates not only with increasing autonomy but also with growing ease of activation. Moreover, this holds for schematic patterns just as it holds for fully substantial types (Langacker 1987: 58). At the level of substantial types, the effect of entrenchment is as described above, with a negative impact on the productivity of the more abstract pattern they instantiate. But at the level of the schema, repeated use and exposure will have the effect of making the schema more easily available by facilitating its activation. Increased availability will in turn facilitate productive use, because the schema will have a greater chance of being retrieved and selected whenever the opportunity for the coining of a neologism arises.

The present findings are mirrored by findings from a corpus-based priming study by AUTHOR (YEAR). Priming is similar to entrenchment in that it boosts the availability of a pattern; it is different in as far as its effect is due to recency rather than frequency and is short-lived rather than more or less stable. The study shows that -ly-derivations are used more creatively (as reflected in an increased type frequency) when -ly-derivation has been primed by a -ly-adverb in the immediately preceding context. Just like a schema that has been primed, a schema that is strongly entrenched will be more available to language users for the creation of new forms than one that is only weakly entrenched, and such greater or lesser availability will result in increased or decreased likelihoods for productive usage. Like priming, then, entrenchment is both a conservative force and a creative force in language (AUTHOR YEAR, YEAR).

The results from the regression analyses are consistent with this double role of entrenchment. Specifically, a pattern with mainly high-frequency types may lose in schematicity and productivity. But a pattern with many types will gain in availability and hence productivity when the pattern also occurs frequently. Plag (2003: 54) elegantly summarizes the relation between morphological productivity, type and token frequency as it has typically been understood:

Unproductive morphological categories will be characterized by a preponderance of words with rather high frequencies and by a small number of words with low frequencies. With regard to productive processes, we expect the opposite, namely large numbers of low-frequency words and small numbers of high-frequency words.

The present findings add one qualification to this view: if the least productive pattern is likely one with only a few high-frequency types, the most productive pattern will be one dominated by an extensive range of infrequent, but not too infrequent types.

\subsection{Understanding individual variation}

The above underscores the value of individual variation studies. The findings support an established theoretical model with new and independent evidence and can even refine that model. The subtler aspects of the interplay between type and token frequency that the analysis of individual variation 
brings to light nicely fit the theory, but would probably have proven harder to unravel using the kinds of evidence that have been typically used so far in productivity studies.

That said, we can also turn the perspective around and ask what the findings tell us about individual variation. Seen from that angle, the present case study highlights several of the practical and theoretical obstacles that individual variation studies must confront. The practical issues should be obvious from the lengthy methodological discussion. They include, among other things, the appropriateness of corpus data, and the operationalization of model variables so that they adhere close enough to the practices of earlier work but can be applied to the current data set. Some healthy scepticism towards the feasibility of corpus-based individual variation studies is not unwarranted. At the same time, this type of study also offers practical advantages, not in the least by the power in numbers and because of their easy replicability.

The practical points aside, however, there is also the promise of new theoretical challenges. The analysis has shown that part of the variation in individual's productivity for a pattern must lie outside the system-internal determinants of productivity we are currently aware of. It is found that individual productivity scores are positively correlated across -ly and -ness-derivation. That this effect, labelled GENERAL PRODUCTIVITY, is consistently retained in the regression models implies that it holds also when the familiar determinants of productivity (a pattern's type and token frequency) are held constant. What is more, GENERAL PRODUCTIVITY does not appear to be explained away by LEXICAL DIVERSITY (which is not retained in the models and with which it shows no strong correlation to begin with), nor by diatypic variation, at least in as far as such variation has been operationalized here through MAIN DESK. At present, then, there is a portion of individual variation that is indeed irreducibly individual. The best that can be made of this is to say that some individuals have a stronger inclination to use patterns productively, irrespective of other known determinants of productivity.

What can explain those remaining individual differences is, for now, food for speculation. Whether an explanation lies in other properties of individuals' linguistic profile, or in more general aspects of individual cognition, or in more strictly language-external factors still remains to be found out. Answers may turn out to be uncomfortable, or trivial. But however it may be, if the study of individual variation is taken seriously such questions need to be addressed.

\section{References}

Baayen, Harald. 1993. On frequency, transparency and productivity. In Geert Booij and Jaap van Marle (eds), Yearbook of morphology 1993, 181-208. Dordrecht: Kluwer.

Baayen, Harald and Rochelle Lieber. 1991. Productivity and word-formation in English: A corpus-based study. Linguistics 29: 801-843.

Barðdal, Jóhanna. 2006. Predicting the productivity of argument structure constructions. Berkeley Linguistics Society 32: 467-478.

Barđdal, Jóhanna. 2008. Productivity: Evidence from case and argument structure in Icelandic. Amsterdam: John Benjamins.

Bolinger, Dwight. 1948. On defining the morpheme. Word 4: 18-23.

Booij, Geert. 2010. Construction morphology. Oxford: Oxford University Press.

Bybee, Joan. 2007. Frequency of use and the organization of language. Oxford: Oxford University Press. Corbin, Danielle. 1987. Morphogie derivationelle et structuration du lexique. Tübingen: Niemeyer. 
Giegerich, Heinz. 2012. The morphology of -ly and the categorial status of 'adverbs' in English. English Language and Linguistics 16: 341-59.

Goldberg, Adele E. 2006. Constructions at work: The nature of generalization in language. Oxford: Oxford University Press.

Gregory, Michael. 1967. Aspects of varieties differentiation. Journal of Linguistics 3: 177-274.

Hay, Jennifer. 2003. Causes and consequences of word structure. London: Routledge.

Hay, Jennifer and Harald Baayen. 2002. Parsing and Productivity. Geert Booij and Jaap van Marle (eds), Yearbook of Morphology 2001, 203-235. Dordrecht: Kluwer.

Langacker, Ronald W. 1987. Foundations of Cognitive Grammar, Vol. 1, Theoretical prerequisites. Stanford: Stanford University Press.

Mollin, Sandra. 2007. The Hansard hazard: Gauging the accuracy of British parliamentary transcripts. Corpora 2: 187-210.

Pinker, Steven. 1999. Words and rules: The ingredients of language. New York:

Plag, Ingo. 1999. Morphological productivity: Structural constraints in English derivation. Berlin: Mouton de Gruyter.

Plag, Ingo. 2003. Word-formation in English. Cambridge: Cambridge University Press.

Rumelhart, David E. and James L. McClelland. 1987. On learning the past tenses of English verbs: Implicit rules or parallel distributed processing? In Brian MacWhinney (ed.), Mechanisms of language acquisition, 195-248. Hillsdale: Lawrence Erlbaum.

Schmid, Hans-Jörg. 2016. A framework for understanding linguistic entrenchment and its psychological foundations. In Hans-Jörg Schmid (ed.), Entrenchment and the psychology of language learning: How we reorganize and adapt linguistic knowledge, 9-35. Berlin: De Gruyter.

Stefanowitsch, Anatol. 2008. Negative entrenchment. A usage-based approach to negative evidence. Cognitive Linguistics 19: 513-531. 
Appendix: Model summaries for $l_{-} \_N Y T A C_{s}$, ness_NYTAC $C_{s}, y_{-} H C_{s}$ and ness_HC $C_{s}$.

\section{Ly_NYTACS}

OLS linear regression

Dependent variable: Productivity

Observations: 697

\begin{tabular}{|c|c|c|c|c|c|}
\hline & Est. & S.E. & t val. & $\mathrm{p}$ & VIF \\
\hline (Intercept) & -0.20 & 0.02 & -8.84 & 0.00 & NA \\
\hline Token frequency & -0.07 & 0.02 & -2.97 & 0.00 & 2.58 \\
\hline Type frequency & 0.52 & 0.03 & 18.40 & 0.00 & 4.19 \\
\hline Main desk 'culture and lifestyle' & 0.30 & 0.06 & 5.16 & 0.00 & 2.37 \\
\hline Main desk 'finance' & 0.15 & 0.04 & 3.44 & 0.00 & 2.37 \\
\hline Main desk 'other' & 0.16 & 0.04 & 4.18 & 0.00 & 2.37 \\
\hline Main desk 'sports' & 0.11 & 0.05 & 2.09 & 0.04 & 2.37 \\
\hline General productivity & 0.24 & 0.02 & 10.41 & 0.00 & 2.75 \\
\hline Token frequency * Type frequency & 0.12 & 0.01 & 14.14 & 0.00 & 1.63 \\
\hline $\begin{array}{l}F(8,688)=511.65, p=0.00 \\
R^{2}=0.86, \text { Adj. } R^{2}=0.85\end{array}$ & & & & & \\
\hline
\end{tabular}

\section{Ness NYTACs}

OLS linear regression

Dependent variable: Productivity

Observations: 696

\begin{tabular}{lrrrrr} 
& Est. & S.E. & t val. & $p$ & VIF \\
\hline (Intercept) & -0.14 & 0.02 & -6.43 & 0.00 & NA \\
Token frequency & -0.12 & 0.03 & -3.48 & 0.00 & 5.66 \\
Type frequency & 0.61 & 0.04 & 15.71 & 0.00 & 8.50 \\
Main desk 'culture and lifestyle' & -0.09 & 0.06 & -1.49 & 0.14 & 2.05 \\
Main desk 'finance' & 0.08 & 0.04 & 1.97 & 0.05 & 2.05 \\
Main desk 'other' & 0.17 & 0.04 & 4.75 & 0.00 & 2.05 \\
Main desk 'sports' & 0.06 & 0.05 & 1.20 & 0.23 & 2.05 \\
General productivity & 0.26 & 0.02 & 10.67 & 0.00 & 3.11 \\
Token frequency * Type frequency & 0.12 & 0.01 & 12.56 & 0.00 & 2.76 \\
\hline $\mathrm{F}(8,687)=608.84, \mathrm{p}=0.00$ & & & & & \\
$\mathrm{R}^{2}=0.88$, Adj. $\mathrm{R}^{2}=0.87$ & & & & & \\
\hline \hline
\end{tabular}




\section{$L y_{-} H C_{S}$}

OLS linear regression

Dependent variable: Productivity

Observations: 171

\begin{tabular}{lrrrrr} 
& Est. & S.E. & t val. & $p$ & VIF \\
\hline (Intercept) & -0.09 & 0.05 & -1.70 & 0.09 & NA \\
Token frequency & -0.03 & 0.07 & -0.45 & 0.65 & 1.80 \\
Type frequency & 0.66 & 0.07 & 9.45 & 0.00 & 2.04 \\
General productivity & 0.24 & 0.05 & 4.48 & 0.00 & 1.23 \\
Token frequency * Type frequency & 0.14 & 0.03 & 4.18 & 0.00 & 1.06 \\
\hline
\end{tabular}

$F(4,166)=62.73, p=0.00$

$\mathrm{R}^{2}=0.60$, Adj. $\mathrm{R}^{2}=0.59$

\section{Ness $H C_{S}$}

OLS linear regression

Dependent variable: Productivity

Observations: 171

\begin{tabular}{lrrrrr} 
& Est. & S.E. & t val. & $p$ & VIF \\
\hline (Intercept) & -0.06 & 0.07 & -0.92 & 0.36 & NA \\
Token frequency & 0.03 & 0.07 & 0.40 & 0.69 & 1.39 \\
Type frequency & 0.37 & 0.09 & 4.25 & 0.00 & 2.03 \\
General productivity & 0.23 & 0.08 & 2.98 & 0.00 & 1.59 \\
Token frequency * Type frequency & 0.12 & 0.05 & 2.31 & 0.02 & 1.11 \\
\hline
\end{tabular}

$F(4,166)=25.12, p=0.00$

$\mathrm{R}^{2}=0.38$, Adj. $\mathrm{R}^{2}=0.36$ 
Wordcount: 8560 words 